\title{
Pediatric Lymphadenopathy: Cytological Diagnosis Over a Period of Two Years in a Rural Teaching Hospital
}

\author{
Manupriya Sharma*, Aruna Gupta and Rashmi Kaul \\ Department of Pathology, Dr. Rajendra Prasad Government Medical College Kangra, Himachal Pradesh, India
}

*Corresponding author: Manupriya Sharma, Assistant Professor, Department of Pathology, Dr. Rajendra Prasad Government Medical College Kangra, Himachal Pradesh, India, Tel: 91-8628000105; E-mail: manupriya.priyasharma@gmail.com

Rec date: October 30, 2017; Acc date: November 12, 2017; Pub date: November 18, 2017

Citation: Sharma M, Gupta A, Kaul R (2017) Pediatric Lymphadenopathy: Cytological Diagnosis Over a Period of Two Years in a Rural Teaching Hospital. Ped Health Res Vol.2: No.3: 16.

\section{Abstract}

Background: Lymphadenopathy (LAP) is a common clinical presentation seen in pediatric patients attending the outpatient department. The etiology of LAP varies from a benign process to malignancy. Fine needle aspiration cytology (FNAC) is a very rapid and easy to perform diagnostic test. Majority of cases can be diagnosed and managed on FNAC without need of histopathology. The aim of this study was to evaluate the role of FNAC in diagnosis of superficial LAP in pediatric population.

Methods: This retrospective study was carried out over a period of two years from January 2015 to December 2016 in the department of Pathology, Dr. RPGMC, Tanda, Himachal Pradesh, India. All cases of superficial LAP seen in pediatric population where FNAC was performed were included in the study.

Results: A total of 357 lymph nodes were aspirated in pediatric population during the study period. Majority of cases (98\%) were benign. The results were categorized into three broad categories as reactive lymphadenitis (296; 82\%), granulomatous lymphadenitis $(56 ; 16 \%)$ and malignancy $(05 ; 02 \%)$. The five cases of malignancies identified in this population comprised of two cases each of Hodgkin's Lymphoma (HL) and Non-Hodgkin's Lymphoma (NHL). One case was diagnosed as metastasis from papillary carcinoma thyroid.

Conclusion: FNAC should be considered as a first line investigation for evaluating the peripheral LAP in pediatric population. The main etiology of LAP is reactive lymphadenitis. Majority of these cases can be diagnosed and managed on FNAC alone. FNAC can play a key role in triage of appropriate cases, so that these patients may be further investigated to make a diagnosis or for referral to higher centers.

Keywords: Fine Needle Aspiration Cytology (FNAC); Pediatric; Lymphadenopathy (LAP); Reactive lymphadenitis; Granulomatous lymphadenitis

\section{Introduction}

Lymphadenopathy (LAP) is a common clinical presentation seen in pediatric population. The etiology for LAP varies from a benign reactive process to infections or malignancies [1]. In majority of the pediatric cases, the enlarged lymph nodes are due to reactive causes and are self-limiting. Hence, neither it is not possible nor desirable to perform excision biopsy in all these cases. Excision biopsy is an invasive procedure requiring anesthesia and it is associated with potential complications [2]. In comparison, FNAC provides a simple, quick, relatively non-invasive and a cost effective diagnostic procedure. This procedure is highly cost effective as it diagnoses majority of cases and helps to triage the selective cases which need further work up and referral to higher centers for management [3-5]. With this approach in mind, FNAC was evaluated retrospectively in diagnoses of LAP in pediatric population.

\section{Materials and Methods}

This retrospective study was carried out over a period of two years from January 2015 to December 2016 in the department of Pathology, Dr. RPGMC, Tanda, Himachal Pradesh, India. It is a tertiary care rural teaching hospital. All pediatric cases ( $<8$ years) with superficial LAP where FNAC was performed during the study period were included. FNAC was done on the representative lymph nodes according to standard protocol and following strict aseptic precautions. An informed consent was taken from all patients before performing the procedure.

\section{Procedure}

All FNAC procedures were performed by the attending pathologists. Relevant clinical details and investigations were recorded. The site and size of the lymph nodes being aspirated was measured. A 22-gauge needle attached to $10 \mathrm{ml}$ syringe mounted on Cameco handle was used for the procedure. The needle was inserted into the swelling and full suction pressure was applied. The needle was moved briskly in different directions in the swelling till a spot of material was visualized in the stem of the needle. The negative pressure of the syringe was released, and the needle was taken out. The needle was removed from the syringe and the aspirated material was 
expelled out on clean glass slides. For smaller lymph nodes $(\leq$ $1 \mathrm{~cm}$ ) non-aspiration technique i.e. needling of the swelling was done. At least 4 good aspirate smears were prepared. Two smears were alcohol fixed and stained with Hematoxylin and Eosin ( $\mathrm{H}$ and $\mathrm{E}$ ) stain and Papanicolaou stain (PAP stain) and another two smears were air dried and stained with Giemsa stain. All cytological smears were evaluated based on adequacy, cellularity, type of cells, arrangement of cells and nuclear as well as cytoplasmic features. Background was evaluated in all smears for any necrosis or inflammatory cells. All cases revealing granulomas/necrosis were further evaluated with Ziehl-Neelson (ZN) staining to rule out any Acid-Fast bacilli (AFB).

\section{Statistical Analysis}

The results were collected and analyzed on a Microsoft Excel 2010 sheet and percentages were calculated.

\section{Results}

\section{Patient characteristics}

A total of 357 cases of pediatric lymph nodes had cytological evaluation during this study period. The age of patients ranged from 4 months to 17 years with mean age of 8 years. The male/female ratio was 2:1.

\section{Broad etiological classification in LAP}

The results were classified into three broad categories: Reactive lymphadenitis (296/357; 82\%), granulomatous lymphadenitis $(56 / 357 ; 16 \%)$ and malignancy $(05 / 357 ; 02 \%)$ as shown in Figure 1. Age-wise distribution of pediatric LAP cases is shown in Figure 2.

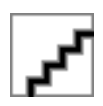

Figure 1 Distribution of pediatric lymphadenopathy cases.

\section{Etiologies based on site}

Cervical lymph node was the main group of lymph nodes enlarged in pediatric population constituting (316/357; 89\%) of all LAP. Majority of the cases of cervical LAP were identified as reactive lymphadenitis $(271 / 316 ; 86 \%)$, followed by granulomatous lymphadenitis $(42 / 316 ; 13 \%)$ and malignancies (03/316; 01\%).

Axillary lymph nodes were the next group of lymph nodes aspirated during our study $(27 / 357 ; 08 \%)$. The main etiology of LAP in these cases was reactive lymphadenitis $(16 / 27 ; 60 \%)$, followed by granulomatous lymphadenitis (11/27; 40\%).

A total of 08 supraclavicular lymph nodes were aspirated in pediatric population in our study. Three cases each (38\%) were identified as reactive lymphadenitis and granulomatous lymphadenitis. Two malignancies (24\%) were identified in pediatric population during this period.

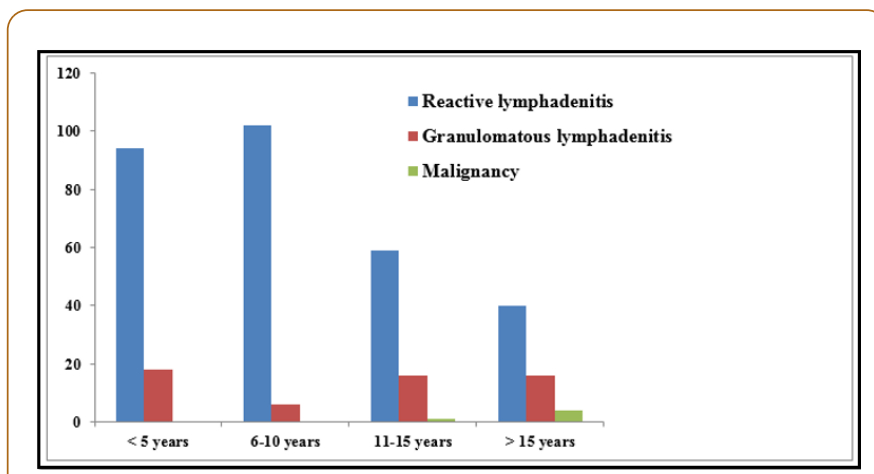

Figure 2 Age-wise distribution of pediatric lymphadenopathy.

A total of 6 inguinal lymph nodes were aspirated during this period and all these lymph nodes revealed reactive lymphadenitis.

Etiologies based A total of 242 lymph nodes were sampled among males in pediatric population. Majority of these cases were reactive lymphadenitis $(210 / 242 ; 87 \%)$, followed by granulomatous lymphadenitis $(28 / 242 ; 11 \%)$ and malignancies (04/242; 02\%).

Among pediatric female population, a total of 115 lymph nodes were aspirated. The main etiology identified in this group was reactive lymphadenitis $(86 / 115 ; 86 \%)$, followed by granulomatous lymphadenitis $(28 / 115 ; 24 \%)$ and malignancies (01/115; 01\%).

\section{Cytological diagnosis: Reactive lymphadenitis}

A total of $296 / 357$ (82\%) cases of reactive lymphadenitis were identified in our study. The cytological features which helped to clinch the diagnosis were cellular smears, polymorphous lymphoid cell population and presence of lympho-histiocytic tangles (Figures $\mathbf{3 a}$ and $\mathbf{3 b}$ ). These cases were followed up. Re-aspiration/excision biopsy was done in cases where the findings did not corroborate with clinical findings, or there was persistence/increase in the size of swelling.

\section{Granulomatous lymphadenitis}

Granulomatous lymphadenitis cases were identified based on the presence of collection of epithelioid cells with or without necrosis (Figure 4a). A total of 56 cases of granulomatous lymphadenitis were identified in this age group. Necrosis was seen in 27 cases. These cases were identified as necrotizing granulomatous lymphadenitis. ZN staining was advised in all 56 cases of granulomatous lymphadenitis. Twelve cases $(21 \%)$ were positive for acid fast bacilli (AFB) and were reported as tubercular lymphadenitis. Rest cases which lacked necrosis and were negative for AFB, were reported as granulomatous lymphadenitis and further clinical work up was advised. 




Figures 3 Reactive lymphadenitis- Smear shows polymorphous lymphoid cell population along with lymph-histiocytic tangles.

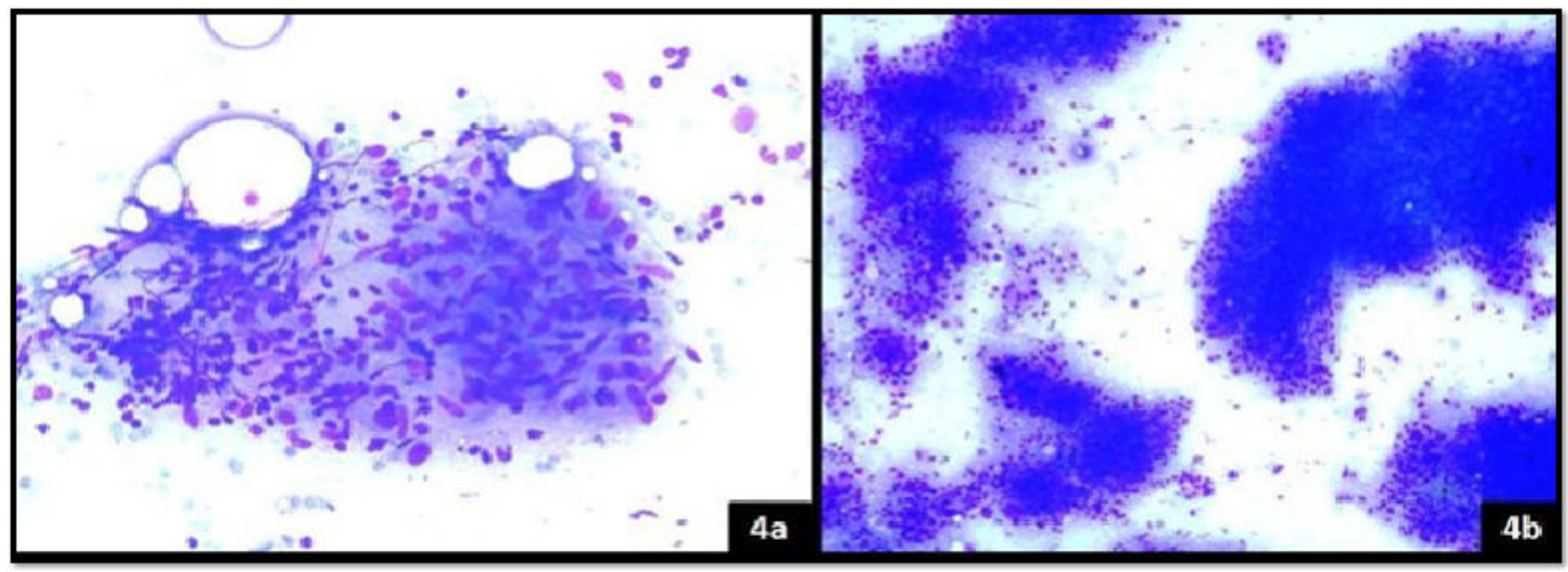

Figure 4 (a)Granulomatous lymphadenitis: Shows well-formed granuloma with reactive lymphoid cells in background. (b) Papillary carcinoma thyroid: Smears show papillary fragments of tumor cells showing overlapping and grooving.

\section{Malignancies}

A total of 5 malignancies were identified during this study period. These cases were classified as: 2 Cases each of Hodgkin's Lymphoma (HL) and Non- Hodgkin's lymphoma (NHL) and one case of papillary carcinoma thyroid. Papillary carcinoma thyroid was identified in one girl of 17 years of age. This case revealed cellular smears with papillary aggregates and sheets of cells. The cells revealed powdery nuclear chromatin, multiple distinct nucleoli, intra-nuclear cytoplasmic inclusions and nuclear grooves (Figure $4 \mathrm{~b}$ ). Two cases of $\mathrm{HL}$ were identified in 12 years and 16 years of age. The cytological features of $\mathrm{HL}$ consisted of scattered typical Reed-Sternberg cells, atypical mononuclear cells in a background of reactive lymphocytes, eosinophils, plasma cells and histiocytes (Figure 5a). Two cases of NHL were identified in 15 years and 17 years of age. Both cases revealed monotonous population of scattered lymphoid cells and background showing lymphoglandular bodies (Figure 5b). All cases of lymphomas diagnosed on cytology were advised excision biopsy of the lymph nodes for histopathological confirmation of diagnosis. Case of papillary carcinoma thyroid was confirmed on histopathology in thyroidectomy specimen. Two cases of NHL and one case of $\mathrm{HL}$ was confirmed on histopathology. These cases were further advised immune-histochemistry (IHC) for characterization of lymphomas. One case of $\mathrm{HL}$ was lost to follow up. 


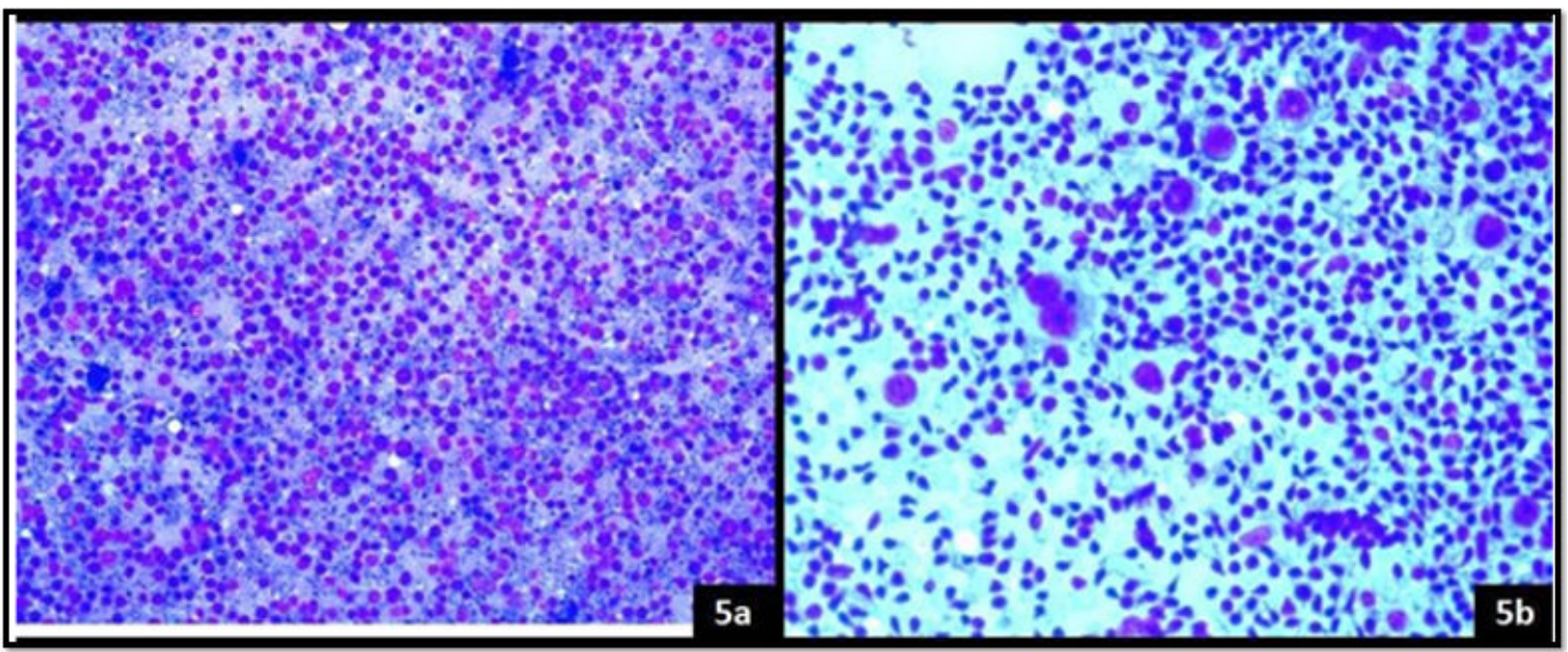

Figure $\mathbf{5}$ (a) NHL: Smear shows monomorphic population of atypical lymphoid cells with lymphoglandular bodies in the background. (b) HL: Smear shows many mononuclear atypical cells and typical Reed Sternberg cells in a background of lymphocytes, eosinophils and plasma cells.

\section{Discussion}

LAP is a common clinical presentation encountered in pediatric population. The etiology in these cases may vary from a benign etiology to a malignant condition. FNAC plays a very important role in evaluating superficial lymph nodes as these are easily accessible for the procedure. Further, FNAC is also advocated due to the ease of the procedure, a relatively non-invasive procedure, quick diagnosis and cost effectiveness [5]. Being the most cost-effective procedure with rapid diagnosis, FNAC is the most frequently used procedure for palpable lesions worldwide.

In this study, a total of 357 cases of superficial lymph nodes were sampled during the study period. In our study, $85 \%$ cases of LAP were due to reactive lymphadenitis and were managed accordingly. Many other studies report comparable results [6-8]. Majority of cases can be diagnosed and managed on FNAC diagnosis alone. However, it is important to clinically correlate the cytological findings. If in any case the cytological diagnosis differs from the clinical diagnosis, excision biopsy and histopathological confirmation of the disease must be considered. Furthermore, excisional biopsy should also be done if the lymph node swelling persists or there is any increase in size of node.

\section{Role of cytology: Benign cases}

Similar to available data, majority of cases were benign (352/357; 98\%) in our study. Only $2 \%$ malignancies were identified in this age group. Among benign cases, 53\% cases were of reactive hyperplasia and $28 \%$ cases of granulomatous lymphadenitis. Characteristic cytological features consisted of polymorphous lymphoid cell population and many tangible body macrophages. Major etiology of LAP in pediatric population in our study was reactive lymphoid hyperplasia (83\%). These cases were followed up for a period of 6 months and majority of these swelling regressed. Marginal cases where the lymph node persisted were excised for histopathological evaluation.

Cytology smears of granulomatous lymphadenitis revealed granulomas, giant cells with or without necrosis. All granulomatous lymphadenitis cases were worked up for AFB (Ziehl-Neelson (ZN) staining). AFB positivity was seen in $21 \%$ cases of granulomatous lymphadenitis. Numerous studies have reported AFB positivity ranging from $10 \%$ to $70 \%$ [9-11]. In countries where TB is endemic, all cases must be worked up for TB until proved otherwise. Presence of Langhan giant cells and caseous necrosis are hallmark of tubercular lymphadenitis [12]. These findings must prompt a pathologist for a detailed work up of the patient for TB. If AFB is not found on ZN staining, culture studies must be advised on aspirate material.

\section{Evaluation of malignancies}

Though only $0.2 \%$ malignancies were identified in pediatric population in our study, it is important to keep these in the differential diagnosis for causes of LAP. All cases identified as malignancies on FNAC, must be confirmed on histopathology.

\section{Conclusion}

The key role of FNAC is to provide a cost effective diagnostic procedure to diagnose majority of cases and to triage appropriate cases which need further work up or referral to higher centers. This will help in avoiding unnecessary invasive procedures in pediatric population and its associated complications. LAP in pediatric population is mainly due to reactive lymphadenitis and is self-limiting. FNAC results must be interpreted by a cyto-pathologist keeping in consideration the clinical, radiological and laboratory findings. 


\section{Disclosure}

The authors declare no conflicts of interest.

\section{References}

1. Twist CJ, Link MP (2002) Assessment of lymphadenopathy in children. Pediatr Clin North Am 49: 1009-1025.

2. Bilal JA, Elshibly EM (2012) Etiology and clinical pattern of cervical lymphadenopathy in Sudanese children. Sudan J Paediatr 12: 97-103.

3. Hirachand S, Lakhey M, Akhter J, Thapa B (2009) Evaluation of fine needle aspiration cytology of lymph nodes in Kathmandu Medical College, Teaching hospital. Kathmandu Univ Med J 7 : 139-142.

4. Ahmed HG, Elmubasher MB, Salih RA, Elhussein GE, Ashankyty IM (2013) Fine needle aspiration cytopathology of pediatric lymphadenopathy among Sudanese children. Asian Pac J Cancer Prev 14: 4359-4363.

5. Hafez NH, Tahoun NS (2011) Reliability of fine needle aspiration cytology (FNAC) as a diagnostic tool in cases of cervical lymphadenopathy. J Egypt Natl Canc Inst 23: 105-114.
6. Adesuwa Olu-Eddo N, Egbagbe EE (2006) Peripheral lymphadenopathy in Nigerian children. Niger J Clin Pract 9: 134-138.

7. Al-Tawfiq JA, Raslan W (2012) The analysis of pathological findings for cervical lymph node biopsies in eastern Saudi Arabia. J Infect Public Health 5: 140-144.

8. Mishra SD, Garg BM (1972) Etiology of cervical lymphadenitis in children. Indian Pediatr 9: 812-815.

9. Lau SK, Wei WU, Hsu C, Engzella UC (1990) Efficacy of fine needle aspiration cytology in the diagnosis of tuberculous cervical lymphadenopathy. J Laryngol Otol 104: 24-27.

10. Dua T, Ahmad P, Vasenwala S, Beg F, Malik A (1996) Correlation of cytomorphology with AFB positivity by smear and culture in tuberculous lymphadenitis. Indian J Tuberc 43: 81-84.

11. Vignesh R, Balakrishnan P, Shankar EM, Murugavel KG, Hanas S, et al. (2007) Value of single acid fast bacilli sputum smears in the diagnosis of tuberculosis in HIV positive subjects. J Med Microbiol 56: 1709-1710.

12. Somu N, Vijayasekaran D, Ravikumar T, Balachandran A, Subramanyam L, et al. (1994) TB disease in pediatrics referral centre. Ind Pediatr 31: 1245-1249. 